\title{
Atividade antimicrobiana de Lippia alba (Mill.) N. E. Brown (Verbenaceae)
}

\author{
Jaciana S. Aguiar, ${ }^{* 1}$ Maria C. C. D. Costa, ${ }^{2}$ Silene C. Nascimento, ${ }^{1}$ Kêsia X. F. R. Sena ${ }^{1}$ \\ ${ }^{1}$ Departamento de Antibióticos, Universidade Federal de Pernambuco, Rua Professor Moraes Rego, s/n, Cidade \\ Universitária, 50670-901 Recife-PE, Brasil, \\ ${ }^{2}$ Departamento de Biologia, Universidade Católica de Pernambuco, Rua do Príncipe, 526, Boa Vista, 50050-900 \\ Recife-PE, Brasil
}

\begin{abstract}
RESUMO: Lippia alba (Mill.) N. E. Brown (Verbenaceae), amplamente distribuída em todo o território brasileiro, é conhecida popularmente como erva cidreira e utilizada na medicina popular como analgésica, febrífuga, antiinflamatória, antigripal e nas afecções hepáticas. Extratos brutos foram preparados a partir de plantas cultivadas, de modo padronizado, em horta medicinal do Laboratório de Fitoterapia da Empresa Pernambucana de Pesquisa Agropecuária (IPA) para a verificação da atividade antimicrobiana, in vitro, pelo método de difusão em disco de papel. A concentração inibitória mínima (CIM) foi determinada para os extratos que exibiram melhores atividades. Os resultados obtidos mostraram que os extratos clorofórmico, acetônico e etanólico da raiz foram ativos frente a Staphylococcus aureus, Micrococcus luteus, Bacillus subtilis, Mycobacterium smegmatis, Candida albicans e Monilia sitophila e os extratos hexânicos, etanólicos e metanólicos das folhas inibiram S. aureus, M. luteus, B. subtilis, M. smegmatis e M. sitophila. A menor concentração inibitória $(\mathrm{CIM}=31,2 \mu \mathrm{g} / \mathrm{mL})$, foi obtida para o extrato clorofórmico da raiz frente a $B$. subtilis e $M$. luteus.
\end{abstract}

Unitermos: Atividade antimicrobiana, Lippia alba, planta medicinal.

\begin{abstract}
Antimicrobial activity of Lippia alba (Mill.) N. E. Brown (Verbenaceae". Lippia alba (Mill.) N. E. Brown (Verbenaceae), commonly known as "erva cidreira", is widely distributed throughout Brazil and is used in folk medicine as an analgesic, anti-inflammatory, cold remedy, as well as to reduce fevers and treat hepatic afflictions. Crude extracts of $L$. alba were prepared from plants cultivated in the medicinal garden of the Laboratório de Fitoterapia of the Empresa Pernambucana de Pesquisa Agropecuária (IPA), State of Pernambuco, Brazil, using standard techniques to test their in vitro antibacterial activity using the paper disk-diffusion method. The minimum inhibitory concentration (MIC) was determined for those extracts demonstrating the highest activity. The results demonstrated that the chloroform, acetone and ethanol extracts of root material were active against the growth of Staphylococcus aureus, Micrococcus luteus, Bacillus subtilis, Mycobacterium smegmatis, Candida albicans and Monilia sitophila, while the hexane, ethanol and methanol extracts of leaves inhibited the growth of $S$. aureus, M. luteus, $B$. subtilis, M. smegmatis and M. sitophila. The lowest inhibitory concentration (MIC $=31.2 \mu \mathrm{g}$ / $\mathrm{mL}$ ) was obtained with the chloroform root extract against B. subtilis and M. luteus.
\end{abstract}

Keywords: Antimicrobial activity, Lippia alba, medicinal plant.

\section{INTRODUÇÃO}

A espécie Lippia alba (Mill.) N. E. Brown pertencente à família Verbenaceae, é um arbusto aromático, cujo aroma está relacionado aos constituintes predominantes nos óleos essenciais, os quais podem variar qualitativamente e quantitativamente, em função de diversos fatores, tais como: estações do ano, época de floração, idade da planta, quantidade de água circulante, resultante da precipitação, fatores geográficos e climáticos (Corrêa, 1992; Matos, 1998; Tavares et al., 2005).

Em 1991 Cáceres et al. citaram pela primeira vez a atividade antimicrobiana dos extratos etanólicos das folhas frente à Staphylococcus aureus; dez anos mais tarde, a ação antimicrobiana de soluções extrativas hidrolcoólicas a $80 \%$ foi verificada frente a este microrganismo (Soares, 2001); Sena Filho et al. (2006) confirmaram atividade antimicrobiana de extratos etanólicos da raiz de L. alba frente a $S$. aureus. Extratos brutos, óleos essenciais e mel do néctar das flores mostraram, atividade antibiótica para diferentes microrganismos (Santos, 1996; Alea et al., 1997; Oliveira, 2000; Rao et al., 2000; Camargo, 2001; Holetz et al., 2002; Pessini et al., 2003; Barbosa, 2003). Mais recentemente, Duarte et al. (2005), referiram a atividade 
antimicrobiana dos óleos essenciais das folhas de $L$. alba sobre Candida albicans.

Além de ações antimicrobianas outras propriedades têm sido atribuídas a $L$. alba quando utilizada na forma de chás, macerada em compressa e banhos. Entre as propriedades atribuídas a espécie destaca-se as ações antiespasmódica, antipirética, antiinflamatória, enemagoga, diaforética, analgésica e sedativa. Tais propriedades devem-se aos seus constituintes ativos, dentre eles o óleo essencial (Julião et al., 2003; Barbosa-Filho et al., 2006).

Considerando a existência de estudos que referem ação antimicrobiana para extratos brutos e óleos essenciais de L. alba, objetivamos neste estudo, comprovar esta ação antimicrobiana para extratos brutos produzidos a partir de plantas cultivadas de forma padronizada para produção de fitoterápicos de modo a confirmar se sob as condições de cultivos em que as plantas estão sendo mantidas os princípios ativos com ação antimicrobiana estão presentes em seus extratos e se atuam com a mesma eficácia referida para outros estudos na inibição dos microrganismos testes.

\section{MATERIAL E MÉTODOS}

\section{Material botânico}

As plantas utilizadas no estudo foram provenientes da horta medicinal do Laboratório de Fitoterapia da Empresa Pernambucana de Pesquisa Agropecuária (IPA), onde as condições de cultivo são controladas e padronizadas de modo a produzir matéria prima utilizada na produção de fitoterápicos. As plantas foram dispostas em duas fileiras, com 20 $\mathrm{cm}$ de espaçamento entre elas, em canteiros medindo aproximadamente, $1 \mathrm{~m}$ de largura por $6 \mathrm{~m}$ de comprimento e $20 \mathrm{~cm}$ de altura e recebendo luz solar pela manhã. $O$ substrato utilizado para o cultivo foi constituído por uma mistura de composto orgânico, húmus de minhoca e barro de jardim (2:1:1) sendo mensalmente acrescido $25 \mathrm{~kg}$ de composto orgânico. A irrigação foi feita por aspersão duas vezes ao dia ( $7 \mathrm{~h}$ às $7 \mathrm{~h}$ e 30 min e $16 \mathrm{~h} 30$ mim às $17 \mathrm{~h}$ ), exceto em períodos chuvosos e a limpeza dos canteiros foi feita a cada sete dias por catação manual.
As coletas do material botânico foram realizadas de novembro a dezembro de 2004. A confirmação da identificação botânica foi realizada pela Dra. Rita de Cássia A. Pereira curadora do Herbário Dárdano de Andrade Lima (IPA), onde um exemplar da espécie foi depositado sob o $\mathrm{n}^{\circ} 61004$.

\section{Preparação dos extratos}

Extratos hexânico, clorofórmico, acetônico, etanólico, metanólico e aquoso de raiz, caule e folhas de $L$. alba foram preparados segundo Zelnik et al. (1977). A extração foi feita por maceração em repouso (oito dias) e agitação (4 h a $200 \mathrm{rpm}$ ) para os extratos aquosos. Em ambos os casos a temperatura de extração esteve entre $28-30{ }^{\circ} \mathrm{C}$ e os extratos após filtrados foram pesados para avaliação dos rendimentos, protegidos da luz e armazenados em geladeira $\left(4^{\circ} \mathrm{C}\right)$. Os extratos alcoólicos foram evaporados a secura e o extrato aquoso foi liofilizado.

\section{Avaliação da atividade antimicrobiana}

A atividade antimicrobiana foi verificada in vitro, pelo método de difusão em disco de papel (Bauer et al., 1966). Os extratos foram testados frente aos seguintes microrganismos que pertencem a coleção de microrganismos do Departamento de Antibióticos da Universidade Federal de Pernambuco: Staphylococcus aureus UFPEDA 01; Bacillus subtilis UFPEDA 16; Enterococcus faecalis UFPEDA 138; Micrococcus luteus UFPEDA 06; Escherichia coli UFPEDA 224; Pseudomonas aeruginosa UFPEDA 39; Serratia marcescens UFPEDA 398; Mycobacterium smegmatis UFPEDA 71; Monilia sitophila UFPEDA 2083; Candida albicans UFPEDA 1007. Os microrganismos teste foram padronizados pela turvação equivalente ao tubo 0,5 da escala de McFarland em solução fisiológica (Barry, 1986; Koneman, 1997), correspondente a uma concentração de aproximadamente $10^{7} \mathrm{UFC} / \mathrm{mL}$ para leveduras e fungos e $10^{8} \mathrm{UFC} / \mathrm{mL}$ para bactérias.

Sobre o meio inoculado, Mueller Hinton ágar (S. aureus, B. subtilis, M. luteus, E. coli, P. aeruiginosa e $S$. marcescens); glicose extrato de levedura ( $E$. faecalis e M. smegmatis) e Sabouraud (C. albicans e

Tabela 1. Rendimento do material botânico de Lippia alba (Mill.) N. E. Brown.

\begin{tabular}{lcccccccc}
\hline \multirow{2}{*}{$\begin{array}{c}\text { Material } \\
\text { Botânico }\end{array}$} & \multicolumn{8}{c}{ Rendimento } \\
\cline { 2 - 9 } & Planta Fresca & $\begin{array}{c}\text { Planta } \\
\text { Seca }\end{array}$ & $\begin{array}{c}\text { Extrato } \\
\text { Bruto }\end{array}$ & EA & EC & EE & EH & EM \\
\hline Raiz & $290 \mathrm{~g}$ & $165 \mathrm{~g}$ & $39,67 \mathrm{~g}$ & $2,69 \mathrm{~g}$ & $2,57 \mathrm{~g}$ & $20,24 \mathrm{~g}$ & $1,76 \mathrm{~g}$ & $12,41 \mathrm{~g}$ \\
Caule & $1060 \mathrm{~g}$ & $475 \mathrm{~g}$ & $39,85 \mathrm{~g}$ & $3,28 \mathrm{~g}$ & $3,16 \mathrm{~g}$ & $6,86 \mathrm{~g}$ & $3,04 \mathrm{~g}$ & $23,51 \mathrm{~g}$ \\
Folha & $1225 \mathrm{~g}$ & $85 \mathrm{~g}$ & $15,61 \mathrm{~g}$ & $1,83 \mathrm{~g}$ & $1,79 \mathrm{~g}$ & $3,48 \mathrm{~g}$ & $1,13 \mathrm{~g}$ & $7,38 \mathrm{~g}$ \\
\hline
\end{tabular}

$\mathrm{EA}=$ Extrato acetônico $; \mathrm{EC}=$ Extrato clorofórmico $; \mathrm{EE}=$ Extrato etanólico; $\mathrm{EH}=$ Extrato hexânico; EM = Extrato metanólico. 
Tabela 2. Atividade antimicrobiana de extratos brutos das folhas de Lippia alba (Mill.) N. E. Brown produzidos a partir da planta seca $(2000 \mu \mathrm{g} / \mathrm{disco})$.

\begin{tabular}{lccccc}
\hline & \multicolumn{3}{c}{ Extratos a $2.000 \mu \mathrm{g} /$ disco } & \multicolumn{2}{c}{ Padrões } \\
\cline { 2 - 6 } Microrganismos & $\mathrm{FH}$ & $\mathrm{FE}$ & $\mathrm{FM}$ & $\begin{array}{c}\text { Kanamicina } \\
(30 \mu \mathrm{g} / \mathrm{disco})\end{array}$ & $\begin{array}{c}\text { Cetoconazol } \\
(300 \mu \mathrm{g} / \mathrm{disco})\end{array}$ \\
\hline S. aureus & $12,0 \pm 0,0$ & $10,3 \pm 1,1$ & $11,3 \pm 2,1$ & 28 & - \\
M. luteus & $20,7 \pm 0,6$ & $24,0 \pm 1,0$ & $25,0 \pm 0,0$ & 34 & - \\
B. subtilis & $15,3 \pm 1,1$ & $14,0 \pm 1,0$ & $13,6 \pm 0,6$ & 29 & - \\
P. aeruginosa & s.a. & s.a. & s.a. & 20 & - \\
M. smegmatis & $13,5 \pm 1,0$ & $13,5 \pm 1,0$ & $13,6 \pm 1,1$ & 40 & - \\
E. faecalis & s.a. & s.a. & s.a. & 13 & - \\
E. coli & s.a. & s.a. & s.a. & 15 & - \\
S. marcescens & s.a. & s.a. & s.a. & 15 & - \\
C. albicans & s.a. & s.a. & s.a. & - & 24 \\
M. sitophila & s.a. & s.a. & $12,0 \pm 0,0$ & - & 22 \\
\hline
\end{tabular}

FH: extrato hexânico da folhas; FE: extrato etanólico da folha; FM: extrato metanólico da folha. sa - sem atividade.

Tabela 3. Atividade antimicrobiana de extratos brutos das raízes de Lippia alba (Mill.) N. E. Brown produzidos a partir da planta $\operatorname{seca}(2000 \mu \mathrm{g} / \mathrm{disco})$.

\begin{tabular}{lccccc}
\hline & \multicolumn{3}{c}{ Extratos a $2.000 \mu \mathrm{g} / \mathrm{disco}$} & \multicolumn{2}{c}{ Padrões } \\
\cline { 2 - 6 } Microrganismos & $\mathrm{RC}$ & $\mathrm{RA}$ & $\mathrm{RE}$ & $\begin{array}{c}\text { Kanamicina } \\
(30 \mu \mathrm{g} / \mathrm{disco})\end{array}$ & $\begin{array}{c}\text { Cetoconazol } \\
(300 \mu \mathrm{g} / \mathrm{disco})\end{array}$ \\
\hline S. aureus & $24,3 \pm 3,0$ & $22,3 \pm 2,3$ & $20,3 \pm 0,6$ & 28 & - \\
M. luteus & $30,0 \pm 0,0$ & $27,0 \pm 1,7$ & $25,7 \pm 2,5$ & 34 & - \\
B. subtilis & $23,3 \pm 0,6$ & $23,3 \pm 0,6$ & $22,0 \pm 1,0$ & 29 & - \\
P. aeruginosa & s.a. & s.a. & s.a. & 20 & - \\
M. smegmatis & $19,3 \pm 1,1$ & $13,7 \pm 1,5$ & $9,3 \pm 1,5$ & 40 & - \\
E. faecalis & s.a. & s.a. & s.a. & 13 & - \\
E. coli & s.a. & s.a. & s.a. & 15 & - \\
S. marcescens & s.a. & s.a. & s.a. & 15 & - \\
C. albicans & $23,0 \pm 1,0$ & $15,6 \pm 0,6$ & $12,8 \pm 0,6$ & - & 24 \\
M. sitophila & $17,3 \pm 1,5$ & s.a. & $15,0 \pm 0,0$ & - & 22 \\
\hline
\end{tabular}

RC: extrato clorofórmico da raiz; RA: extrato acetônico da raiz; RE: extrato etanólico da raiz.; sa - sem atividade.

Tabela 4. Valores das concentrações inibitórias mínimas (CIM) em $\mu \mathrm{g} / \mathrm{mL}$ para os extratos brutos de raiz e folha de Lippia alba (Mill.) N. E. Brown frente aos microrganismos testes.

\begin{tabular}{lcccccc}
\hline \multirow{2}{*}{ Microrganismos } & \multicolumn{7}{c}{ Extratos de planta seca $(\mu \mathrm{g} / \mathrm{mL})$} \\
\cline { 2 - 7 } & FH & FE & FM & RC & RA & RE \\
\hline S. aureus & n.t. & n.t. & n.t. & 500 & 250 & 1000 \\
B. subtilis & 500 & 62,5 & 250 & 31,2 & 125 & 125 \\
M. smegmatis & n.t. & n.t. & n.t. & 31,2 & 250 & 250 \\
C. albicans & n.t. & n.t. & n.t. & n.t. & n.t. & n.t. \\
M. sitophila & n.t. & n.t. & n.t. & 2000 & 2000 & n.t. \\
\hline
\end{tabular}

Extratos: FH, FE, FM - Folha hexano, etanol, metanol; RC, RA, RE - Raiz clorofórmio, acetona, etanol. nt - não testado. 
M. stophila), foram colocados discos de papel xarope estéreis, de $6 \mathrm{~mm}$ de diâmetro, embebidos com $10 \mu \mathrm{L}$ da solução do extrato a $200.000 \mu \mathrm{g} / \mathrm{mL}$. Após a colocação dos discos, as placas foram incubadas durante $24 \mathrm{~h}$ e 48 $\mathrm{h}$ à temperatura de $30^{\circ} \mathrm{C}$ e $37^{\circ} \mathrm{C}$.

Todos os extratos brutos de L. alba foram testados frente às bactérias Gram-positivas, Gramnegativas, álcool-ácido resistente, levedura e fungo filamentoso. Os extratos que apresentaram boa atividade antimicrobiana foram retestados em triplicata e os diâmetros dos halos foram expressos pela média \pm desvio padrão dos resultados obtidos nas três repetições. Halos iguais e superiores a $10 \mathrm{~mm}$ foram considerados significativos de atividade antibiótica (Awadh Ali et al., 2001; Bakshu et al., 2001; Khan et al., 2001 e Chowdhury et al., 2002; Ferronatto et al., 2007; Silva et al., 2007; Nascimento et al., 2007).

Os antibióticos kanamicina e cetoconazol foram utilizados no testes como padrões, nas concentrações de $30 \mu \mathrm{g} /$ disco e $300 \mu \mathrm{g} /$ disco, respectivamente.

A concentração inibitória mínima (CIM) foi determinada, para os extratos que exibiram melhores atividades antibióticas, pelo método de diluições seriada em meio sólido (Carvalho et al. 2002). Alíquotas de diferentes volumes $(1,0,0,5,0,25,0,125,0,06$ e 0,03 $\mathrm{mL}$ ) de uma solução a $20.000 \mu \mathrm{g} / \mathrm{mL}$ foram colocadas em placa de Petri e homogeneizadas com $10 \mathrm{~mL}$ do meio de cultura ideal para o crescimento de cada microrganismo. Os microrganismos teste foram semeados, em estrias, sobre a superfície do meio e as placas foram incubadas à $37^{\circ} \mathrm{C}$ e $30^{\circ} \mathrm{C}$ por $24 \mathrm{~h}$ e $48 \mathrm{~h}$. Valores de CIM iguais ou superiores a $1000 \mu \mathrm{g} / \mathrm{mL}$ não foram considerados satisfatórios (Holetz et al. 2002).

\section{RESULTADOS E DISCUSSÃO}

O material botânico coletado (raiz, caule e folha), após a extração mostrou que a raiz de $L$. alba teve o melhor rendimento de extratos brutos, em relação ao caule e a folha. Considerando-se os solventes utilizados nas extrações das folhas e dos caules, extrato metanólico apresentou o melhor rendimento que os extratos acetônico, clorofórmico, etanólico e hexânico e para as raízes o extrato etanólico apresentou o melhor rendimento que os extratos acetônico, clorofórmico, metanólico e hexânico (Tabela 1).

Extratos hexânicos, etanólicos e metanólicos das folhas de $L$. alba apresentaram atividade frente a $S$. aureus, M. luteus, B. subtilis, M. smegmatis e $M$. sitophila, sendo os melhores resultados observados para os extratos hexânico, etanólico e metanólico frente a M. luteus $(20,7 \pm 0,6 \mathrm{~mm}, 24,0 \pm 1,0 \mathrm{~mm}$ e 25,0 $\pm 0,0$ $\mathrm{mm}$ respectivamente) e extrato metanólico frente a $M$. sitophila $(12,0 \pm 0,0 \mathrm{~mm})$ (Tabela 2).

Extratos clorofórmico, acetônico e etanólico das raízes de $L$. alba foram ativos frente a $S$. aureus,
M. luteus, B. subtilis, M. smegmatis, C. albicans e $M$. sitophila sendo os melhores resultados observados para os extratos clorofórmico, acetônico e etanólico frente a $S$. aureus $(24,3 \pm 3,0 \mathrm{~mm}, 22,3 \pm 2,3 \mathrm{~mm}$ e $20,3 \pm 0,6$ $\mathrm{mm}$ respectivamente), $M$. luteus $(30,0 \pm 0,0 \mathrm{~mm}, 27,0 \pm$ 1,7 e $25,7 \pm 2,5 \mathrm{~mm}$ respectivamente), B. subtilis $(23,3$ $\pm 0,6 \mathrm{~mm}, 23,3 \pm 0,6 \mathrm{~mm}$ e $22,0 \pm 1,0$ respectivamente); extratos clorofórmico e acetônico frente a $C$. albicans $(23,0 \pm 1,0 \mathrm{~mm}$ e $15,6 \pm 0,6 \mathrm{~mm}$ respectivamente); extratos clorofórmico e etanólico frente a $M$. sitophila $(17,3 \pm 1,5 \mathrm{~mm}$ e $15,0 \mathrm{~mm}$ respectivamente). Nenhum extrato mostrou atividade frente a E. faecalis, E. coli, $P$. aeruginosa e $S$. marcescens (Tabela 3 ).

A atividade antimicrobiana de $L$. alba, principalmente frente a bactérias Gram-positivas verificada no presente estudo, havia sido constatada para o óleo essencial da espécie frente a $B$. subitilis e $E$. faecalis (Alea et al., 1997), para os extratos etanólicos, hidroalcoólicos das folhas a $80 \%$ e extratos etanólico da raiz, frente a $S$. aureus (Cáceres et al., 1991; Soares, 2001; Sena Filho et al., 2006). Nenhuma publicação referiu ação antimicrobiana de extratos brutos frente a $M$. luteus, B. subtilis, C. albicans e M. sitophila, sendo, portanto, estudo o primeiro a verificar a ação de extratos brutos da espécie para estes microrganismos. A atividade antimicrobiana observada no nosso estudo para extratos brutos de L. alba, demonstrou que nas condições de cultivo em que as plantas estão sendo mantidas, princípios ativos com ação antimicrobiana estiveram presentes, sobretudo nas raízes, cujos extratos brutos inibiram o crescimento de $S$. aureus, M. luteus, B. subtilis, C. albicans e M. sitophila, com halos de inibição com diâmetros próximos daqueles produzidos pelos antibióticos padrões para estes microrganismos.

As atividades antifúngicas dos extratos clorofórmico e acetônico da raiz verificadas em nossos estudos, confirmam os estudos de Santos (1996) e Oliveira (2000) e Rao et al. (2000), que comprovaram a ação antifúngica para extratos brutos das folhas de $L$. alba e para seus óleos essenciais.

A avaliação da concentração inibitória mínima dos extratos das folhas e raízes de $L$. alba, mostraram concentrações inibitórias entre 31,2 a $1000 \mu \mathrm{g} / \mathrm{mL}$ para $S$. aureus e B. subtilis (Tabela 4), diferindo dos resultados mencionados por Holetz et al. (2002) e Pessini et al. (2003), os quais encontraram valores de CIM para extratos hidroalcoólicos das folhas e das partes aéreas de L. alba superiores $1000 \mu \mathrm{g} / \mathrm{mL}$ frente a estes microrganismos; diferindo também dos resultados encontrados por Sena Filho et al. (2006) que observaram valores para extratos etanólicos das raízes iguais e superiores a $2 \mathrm{mg} / \mathrm{mL}$ frente a $S$. aureus.

A atividade antimicrobiana observada para os extratos das raízes e das folhas de L. alba incentivam novas pesquisas com substâncias isoladas com o intuito de estabelecer o constituinte ou os constituintes químicos responsáveis por tal atividade. 


\section{REFERÊNCIAS}

Alea JA, Luis AG, Peréz AR, Jorge MR, Baluja R 1997. Composición y propiedades antibacterianas del aceite esencial de Lippia alba (Mill.) N. E. Br. Rev Cubana Farm 30: 29-35.

Awadh Ali NA, Julich WD, Kusnick C, Lindequist U 2001. Screening of Yemeni medicinal plants for antibacterial and cytotoxic activities. J Ethnopharmacol 74: 173179

Bakshu LMD, Ram AJ, Raju RRV 2001. Antimicrobial activity of Securinega leucopyrus. Fitoterapia 72: 930-933.

Barbosa FG 2003. Contribuição ao conhecimento químico de três quimiotipos de Lippia alba (Mill.) N. E. Brown, cultivados no horto de plantas medicinais da UFC. 280p. Tese de Doutorado em Química Orgânica, Universidade Federal do Ceará.

Barbosa-Filho JM, Medeiros KCP, Diniz MFFM, Batista LM, Athayde-Filho PF, Silva MS, Cunha EVL, Almeida JRGS, Quintans-Júnior LJ 2006. Natural products inhibitors of the enzyme acetylcholinesterase. Rev Bras Farmacogn 16: 258-285.

Barry AL 1986. Procedure for testing antimicrobial agents in agar media: theoretical considerations. In: Lorian $\mathrm{V}$ (ed) Antibiotics in Laboratory Medicine. Baltimore: Williams \& Wilkins. p.13.

Bauer AW, Kirby WMM, Sherris JC, Turk M 1966. Antibiotic susceptibility testing by the standardized single disk method. Am J Clin Pathol 45: 493 - 496.

Cáceres A, Alvarez AV, Ovando AEO, Samayoa BE 1991. Plants used in Guatemala for the treatment of respiratory diseases. 1 . Screening of 68 plants against gram-positive bacteria. $J$ Ethnopharmacol 31: $193-$ 208.

Camargo RCR 2001. Biologia Floral da Lippia alba (Verbenaceae), atividade antibacteriana $e$ caracterização de amostras de méis de Apis mellifera L. Botucatu, 99p. Tese de Doutorado em Zootecnia Nutrição e produção animal, Universidade Estadual Paulista Júlio de Mesquita Filho.

Carvalho AA, Sampaio MCC, Sampaio FC, Melo AFM, Sena KXFR, Chiappeta AA, Higino JS 2002. Atividade antimicrobiana in vitro de extratos hidroalcoólico de Psidium guajava L. sobre bactérias Gram-negativas. Acta Farm Bonaerense 21: 255-258.

Chowdhury D, Sayeed A, Islam A, Bhuiyan MSA, Khan GRMAM 2002. Antimicrobial activity and cytotoxicity of Aerva lanata. Fitoterapia 73: 92-94.

Corrêa CBV 1992. Contribuição ao estudo de Lippia alba (Mill.) N. E. Br. ex Britt \& Wilson - erva-cidreira Rev Bras Farm 73: 57-64.

Duarte MCT, Figueira GM, Sartoratto A, Rehder VLG, Delarmelina C 2005. Anti-Candida activity of Brazilian medicinal plants. J Ethnopharmacol 97: 305-311.

Ferronatto R, Marchesan ED, Pezenti E, Bednarski F, Onofre SB 2007. Atividade antimicrobiana de óleos essenciais produzidos por Baccharis dracunculifolia D.C. e Baccharis uncinella D.C. (Asteraceae). Rev Bras Farmacogn 17: 224-230.

Holetz FB, Pessini GL, Sanches NR, Cortez DAG, Nakamura CV, Dias Filho BP 2002. Screening of some plants used in the Brazilian folk medicine for the treatment of infectious diseases. Mem Inst Oswaldo Cruz 97: 1027-1031.

Julião LS, Tavares ES, Lage CLS, Leitão SG 2003 Cromatografia em camada fina de extratos de três quimiotipos de Lippia alba (Mill) N. E. Br. (erva cidreira). Rev Bras Farmacogn 13: 36-38.

Khan MR, Kihara M, Omoloso AD 2001. Antimicrobial activity of Symplocos cochinensis. Fitoterapia 72: 825-828.

Koneman EW 1997. Diagnóstico microbiológico. México: J. B. Lippincott Co.

Matos FJA 1998. Farmácias vivas. Fortaleza: EUFC.

Nascimento PFC, Nascimento AC, Rodrigues CS, Antoniolli AR, Santos PO, Barbosa Júnior, Trindade RC 2007. Atividade antimicrobiana dos óleos essenciais: uma abordagem multifatorial dos métodos. Rev Bras Farmacogn 17: 108-113.

Oliveira AC 2000. Atividade de óleos essenciais e exsudatos de plantas sobre espécies fúngicas isoladas de frutas in natura. João Pessoa, 86p. Dissertação de Mestrado em Ciência e Tecnologia dos alimentos Universidade Federal da Paraíba.

Pessini GL, Holetz FB, Sanches NR, Cortez DAG, Dias-Filho BP, Nakamura CV 2003. Avaliação da atividade antibacteriana e antifúngica de extratos de plantas utilizados na medicina popular. Rev Bras Farmacogn 13 (Supl. 1): 21-24.

Rao GP, Singh M, Singh P, Catalan C, Kapoor IPS, Singh OP, Singh G 2000. Studies on chemical constituents and antifungal activity of leaf oil of Lippia alba (Mill). Indian J Chem Technol 7: 332-335.

Santos MMFB 1996. Efeito de extratos de duas formas de Lippia alba sobre o fungo Colletotrichum glocosporioides (Penz.) isolado de Citrus sp. São Paulo, 105p. Dissertação de Mestrado em Fisiologia e Bioquímica de plantas - Escola Superior de Agricultura Luiz Queiroz, Universidade de São Paulo

Sena Filho JG, Melo JGS, Saraiva AM, Gonçalves AM, Psiottano MNC, Xavier HS 2006. Antimicrobial activity and phytochemical prolife from the roots of Lippia alba (Mill.) N. E. Brown. Rev Bras Farmacogn 16: 506-509.

Silva JG, Souza IA, Higino JS, Siqueira-Junior JP, Pereira JV, Pereira MSV 2007. Atividade antimicrobiana do extrato de Anacardium occidentale Linn. em amostras multiresistentes de Staphylococcus aureus. Rev Bras Farmacogn 17: 572-577.

Soares L 2001. Estudo tecnológico, fitoquímico e biológico de Lippia alba (Miller) N. E. Brown ex Britt. \& Wils. (falsa melissa) Vernbenaceae. 112p. Dissertação de Mestrado em Farmácia - Universidade Federal de Santa Catarina.

Tavares ES, Julião LS, Lopes D, Bizzo HR, Lage CLS, Leitão SG 2005. Análise do óleo essencial de folhas de três quimiotipos de Lippia alba (Mill.) N.E.Br. (Verbenaceae) cultivados em condições semelhantes. Rev Bras Farmacogn 15: 1-5.

Zelnik RD, Lavie EC, LevyAHJ, Wang Paul IC 1977. Barbatusin and cyclobutatusin, two novel diterpenoides from Coleus barbatus Benthan. Tetrahedron 33: 14571467. 